\title{
Modelo de gestión de la investigación para incrementar la producción científica de los docentes universitarios del Perú
}

\section{Research management model to increase the scientific production of university teachers in Perú}

Miguel Angel Valles-Coral1

Recibido: abril 28 de 2019

Aceptado: junio 28 de 2019

\section{Resumen}

La universidad peruana atraviesa un profundo proceso de cambios que ameritan nuevos modelos de gestión universitaria, orientados a la búsqueda de soluciones y al incremento de la producción científica. El objetivo fue diseñar un modelo de gestión de la investigación, basado en la teoría del modelo de promoción de la investigación, de manera que se incremente la producción científica de los docentes. Se realizó una investigación no experimental, descriptiva propositiva, con una muestra de 62 docentes que realizan investigación, a quienes se aplicó un cuestionario para recoger datos sobre la problemática. El $87 \%$ de los encuestados considera que la gestión de la investigación es mala o regular, mientras que el $91 \%$ considera que no se hace nada por la producción científica. Se concluye con el diseño de un nuevo modelo de gestión, basado en tres pilares: institucionalidad, para garantizar la adopción del modelo en la universidad; gestión de la investigación propiamente dicha, que busca fortalecer las competencias para la publicación de artículos científicos; y gestión administrativa, para asegurar las condiciones logísticas y operativas en la ejecución de los proyectos de investigación.

\begin{abstract}
The Peruvian university is going through a deep process of changes that merit new models of university management, aimed at finding solutions and increasing scientific production. A non-experimental, descriptive purposeful, investigation was carried out, with a sample of 62 teachers conducting research, to whom a questionnaire was applied to collect data on the problem. $87 \%$ of the respondents consider that the management of the research is bad or regular; while $91 \%$ consider that nothing is done for the scientific production. It concludes with the design of a new management model, based on three pillars: institutionality, to guarantee the adoption of the model in the university; management of the research itself, which seeks to strengthen the competences for the publication of scientific articles; and administrative management, to ensure the logistical and operational conditions in the execution of research projects.
\end{abstract}

Keywords: models, research, university management, scientific production.

Palabras clave: modelos, investigación, gestión universitaria, producción científica.

1 Ingeniero de sistemas, doctorando en Gestión Pública y Gobernabilidad, magíster en Administración de Negocios y Relaciones Internacionales, Universidad Nacional de San Martín, Tarapoto, Perú. E-mail: mavalles@unsm.edu.pe ORCID: 0000-0002-8806-2892 


\section{Introducción}

La reforma de Córdoba, ocurrida en 1918, criticaba el carácter elitista y aristocrático con el que venían funcionando las universidades en el cono sur (Arias-Gallegos, 2013), y buscaba democratizar la universidad, dotándola de un carácter más científico y humanista. Desde entonces, la promulgación en el Perú de la Ley Universitaria (Ley 30220, 2014), representa un importante punto de inflexión y cambios, a partir de la cual se replantearon en términos de calidad y cantidad de la producción científica, la forma en que se gestionan las universidades (British Council, 2016). Se trata del inicio de un nuevo sistema universitario, dado que la calidad de la educación superior de un país es uno de los fundamentos de su desarrollo (Lavalle $\&$ de Nicolas, 2017).

En ese sentido, Cuencia (2014), nos llama a tomar esta oportunidad de cambio, para reflexionar acerca de cuáles son los retos que debe enfrentar el nuevo modelo de gobernanza universitaria, y hacia donde debería dirigirse el desarrollo de la educación superior en el Perú. Asimismo, plantea reconstruir la relación que la universidad sostuvo en sus inicios con la sociedad, actuando como agente democratizador e igualador, reafirmando el papel que juega en la comprensión y reconstrucción de la identidad nacional. Estos deberían ser componentes fundamentales en la misión universitaria, pues la universidad busca no solo el crecimiento económico, sino el desarrollo socioeconómico nacional, objetivo difícilmente alcanzable si persisten las grandes diferencias económicas y sociales del Perú.

Lo anterior implica la necesidad de hacer profundos cambios a la estructura actual de gestión de la investigación de las universidades nacionales, por modelos de licenciamiento que exijan el cumplimiento de estándares mínimos de funcionamiento y acreditación, basados en resultados palpables y medibles, que calcen en un esquema de mejora continua. Esto permitirá que la universidad logre el papel que le ha sido asignado en el desarrollo del país, a través del perfeccionamiento de la gestión universitaria de cada uno de los procesos que la integran (Acosta, Becerra \& Jaramillo, 2017). Una educación superior de calidad y pertinencia, que provea de técnicos y profesionales competitivos al país, es clave para sostener el proceso de desarrollo económico y social, en esta era del conocimiento (SINEACE, 2013).

En ese contexto, un reclamo recurrente a la universidad peruana es su desconexión con la realidad del país y el casi nulo aporte a la solución de sus problemas. Esto debido en parte a: la desigual calidad de la educación, la desconexión con las necesidades del sector productivo, la limitada oferta de carreras profesionales y los escasos resultados de su producción científica. Como lo indican Martínez-de Ita, Piñero y Figueroa-Delgado (2013):

(...) La relación que la universidad guarda con el desarrollo ha sido objeto de una variedad tan grande de interpretaciones como perspectivas teóricas sobre la sociedad existen. También las sucesivas etapas del desarrollo social condicionan los conceptos sobre estas instituciones, y así se modifican también las nociones sobre su relación con el desarrollo".

En el caso de las universidades estatales peruanas, los productos resultantes de la investigación, no se articulan con las funciones de: generación de conocimiento para la producción; la formación de fuerza de trabajo altamente calificada; la generación de los cuadros para la conducción económica, social y política de los países; y la producción de ideología, incluida la crítica, orientada al mejoramiento del estado de cosas en la sociedad (Martínez-de Ita et al., 2013).

En este sentido, la nueva ley es una oportunidad para reestructurar los modelos de gestión universitarios, que involucre a todas las unidades y personas, de forma individual o colectiva, y asuma decididamente el reto de la calidad (Universidad Politécnica de Cataluña \& Centro Interuniversitario de Desarrollo, 2007). La articulación de los ac- 
tores del proceso investigativo, permitirá una mejor gestión de la investigación, con mayor y mejor producción científica, publicada, visible y con factores de impacto que califique sus resultados. Esto permitirá la adaptación a los cambios socioeconómicos ocurridos en las dos últimas décadas, que integren el desarrollo económico con la calidad universitaria (Jiménez-Tello, 2007).

Para Cáceres-Castellanos (2014), la divulgación de la investigación científica, mediante artículos, ponencias y conferencias, entre otros mecanismos, es esencial, pues, como comúnmente se reconoce, investigación que no se publica no existe. Se puede decir que la investigación culmina al ser publicada en una revista científica; solo así será conocida por la comunidad académica, sus resultados serán discutidos y su contribución hará parte del conocimiento científico universal.

En este escenario, el problema aquí abordado trata sobre la Universidad Nacional de San Martín Tarapoto, UNSM-T, y los ajustes que deberían efectuarse para alcanzar la misión de: ser el espacio de formación de pensamiento crítico, desarrollo de la investigación e innovación, buscando la solución de los problemas de la sociedad. Para ello, la UNSM-T ha sido una de las primeras en adecuarse a la nueva Ley Universitaria (Gestion, 2015). Ese proceso ha generado la reformulación de sus principales documentos normativos, a saber: estatutos, reglamentos, normas y directivas, encaminadas todas a lograr el cumplimiento de las condiciones básicas de calidad, así como el licenciamiento, en un tiempo razonable (Suárez \& Díaz, 2014). Estos cambios tienen como razón principal tres aspectos importantes: formación académica, extensión universitaria y proyección social e investigación, lo que obliga a realizar investigación y publicar para garantizar una adecuada visibilidad de la producción científica.

En vista de lo anterior, se plantea como objetivo del presente trabajo la creación de un modelo de gestión de la investigación basado en la teoría del modelo de promoción de la investigación, que ar- ticule los engranajes de la investigación en la universidad, de manera que: i) se terminen de revisar y reformular los documentos normativos relacionados con la investigación; ii) a través de un proceso participativo, se socialicen, acepten y adopten estos documentos; iii) se promueva una mayor participación en investigación por parte de los docentes y estudiantes de la universidad; iv) se fortalezcan competencias integrales en los docentes para la redacción y publicación de artículos científicos; v) que los resultados de las investigaciones puedan condensarse en artículos científicos para ser sometidos y posteriormente publicados en revistas científicas; vi) se trabaje articuladamente con una propuesta de identidad digital del investigador, para lograr una mayor visibilidad de la producción científica; vii) se reconozca a los docentes que se comprometen con el logro de productos relacionados a este modelo de gestión de la investigación; viii) se adopte la propuesta como un modelo de gestión de la investigación y la visibilidad para incrementar el ranking de la producción científica; de manera que se incremente la producción científica de los docentes universitarios.

\section{Marco teórico y métodos}

\subsection{Situación actual de la universidad}

La aparición de los rankings internacionales, ha catapultado a las universidades de investigación como referentes para la calidad universitaria. El gran peso que estas clasificaciones dan a la investigación, ha llevado a que las universidades que aparecen en los primeros lugares, sean instituciones donde la investigación desempeña un papel importante en sus procesos (Lavalle $\&$ de Nicolas, 2017).

Existen diferentes rankings, como el de Webometrics, que evalúan la cantidad y calidad de las publicaciones (Aguillo, Ortega \& Fernández, 2008). La UNSM-T, a Julio de 2018 en Webometrics, a nivel de universidades peruanas, se encuentra ubicada en el puesto 64 de 182 entidades con rango universitario (Aguillo, 2018). Los motivos que han llevado 
a que la universidad no esté bien posicionada en el ranking son estructurales, como: falta de personal, infraestructura y equipamiento para la investigación, la falta de financiamiento $y$, sobre todo, la "ausencia de un clima intelectual", que ofrezca apoyo a la investigación (Ortiz-Torres, 2018).

Ejemplo de ello es que, aún con recursos insuficientes, entre los años 2014 a 2017, en promedio se financiaron 50 proyectos de investigación por año. Los resultados de estos proyectos, se encuentran en informes finales que reposan en la biblioteca general de la universidad, pero muy pocos han sido publicados en revistas científicas. En otras palabras, como lo menciona Cáceres-Castellanos (2014), esos trabajos "simplemente no existen para efectos de calificación y posicionamiento de la universidad". Los resultados de la investigación deben divulgarse entre quienes pueden beneficiarse de los mismos (Vargas, 2019). Para ello deben implementarse mecanismos de vigilancia de la originalidad, a través de sistemas de evaluación del impacto de las investigaciones (Valles-Coral \& Rengifo-Arias, 2018).

Otro elemento que agudiza el problema, es la deficiente articulación entre el eje estratégico, el táctico y el operativo. Eje estratégico representado por el vicerrectorado de investigación y sus documentos de gestión, que todavía siguen en proceso de reformulación y maduración. El eje táctico, que incluye a la Oficina de Investigación y a las unidades de investigación de la universidad encargados de: estandarizar y socializar procedimientos de investigación; promover la creación de revistas indexadas, junto con la publicación de los resultados de investigación. Asimismo, el eje operativo, representado por la comunidad universitaria, involucrada en: la identificación, formulación y ejecución de proyectos de investigación, así como la síntesis de sus resultados en artículos científicos de alto impacto.

Para revertir esta situación, acorde con (Monteza-Arbulú, 2014), son necesarios cambios estructurales que invitan a repensar la idea de universidad, permisible ahora gracias a la nueva ley universitaria. Se trata de gestionar la investigación universitaria para responder a las demandas del entorno, a través de la búsqueda del conocimiento, su transmisión y la articulación de las tres funciones básicas de la universidad: Investigación, docencia y extensión (Suárez \& Díaz, 2014; Forero-Jiménez, 2019).

\subsection{Metodología}

Este trabajo de investigación emplea un diseño no experimental, longitudinal, descriptivo propositivo, y de corte cuantitativo. La población estuvo conformada por todos los docentes de la Universidad Nacional de San Martín - Tarapoto (UNSM-T), que a febrero de 2018 era un total de 530 docentes. La muestra se determinó aplicando la fórmula de muestreo con población finita conocida, resultando 62 docentes, con un nivel de confianza del $95 \%$ y margen de error de $12 \%$.

El diseño de los instrumentos utilizados partió de propuestas como las de Suárez y Díaz (2014), quienes dimensionan la gestión de la investigación de la siguiente manera: objetivos y estrategias; prácticas directivas, estructura y organización, métodos, instrumentos y herramientas, y cultura y personalidad. También se tuvo en cuenta el estudio de Acosta, Becerra y Jaramillo (2017), que considera prioritaria la incorporación del componente de tecnología de información y comunicaciones; así como la investigación de Monteza-Arbulú (2014), quien de manera interesante propone elevar la pertinencia de los trabajos de investigación de los docentes, al igual que las tesis de los graduados. El principal referente fue el modelo de Promoción de la Investigación de la Pontificia Universidad Católica (Chávez-Rodríguez, 2016), ya que esta institución se encuentra muy bien posicionada en diversos rankings nacionales e internacionales (SUNEDU, 2018; Aguillo, 2018; Times Higher Education, 2019).

La información se recopiló a través de dos instrumentos: i) encuesta sobre la situación actual del 
sistema de gestión de la investigación, que cuenta con 7 dimensiones y 24 indicadores, con escala dicotómica; ii) Encuesta sobre el estado actual de la producción científica, que cuenta con 7 dimensiones y 27 indicadores, en escala de Likert. Estos instrumentos pasaron por una prueba de confiabilidad mediante un piloto, con prueba de Kuder Richardson igual a 0,9238 y alfa de cronbach igual a 0.9087 , con validez mediante juicio de expertos de 4.84 y 4.80 , respectivamente, en una escala de 1 a 5.

En una primera fase se realizó un diagnóstico de la gestión de la investigación, características principales, recursos humanos y logísticos, procesos involucrados y componentes de tecnología de información que se necesitan incorporar. En paralelo, se revisaron los modelos de gestión de la investigación que usan las universidades mejor posicionadas en el Perú, para proponer un nuevo modelo que se adecúe mejor a la realidad. También se realizó el diagnóstico de la situación actual de la producción científica de la Universidad, mediante métodos bibliométricos. Los datos fueron recopilados en una base de datos y procesados mediante spss v.24.

\section{Resultados y discusión}

\subsection{La gestión de la investigación}

En la figura 1 se observa que en la dimensión de políticas institucionales de promoción, un $31 \%$ de los docentes considera que se realiza buena gestión. Sin embargo, en las otras dimensiones, entre el $11 \%$ y $13 \%$ consideran buena la gestión de los mismos, mientras que solo un porcentaje muy bajo considera el seguimiento de los procesos como bueno.

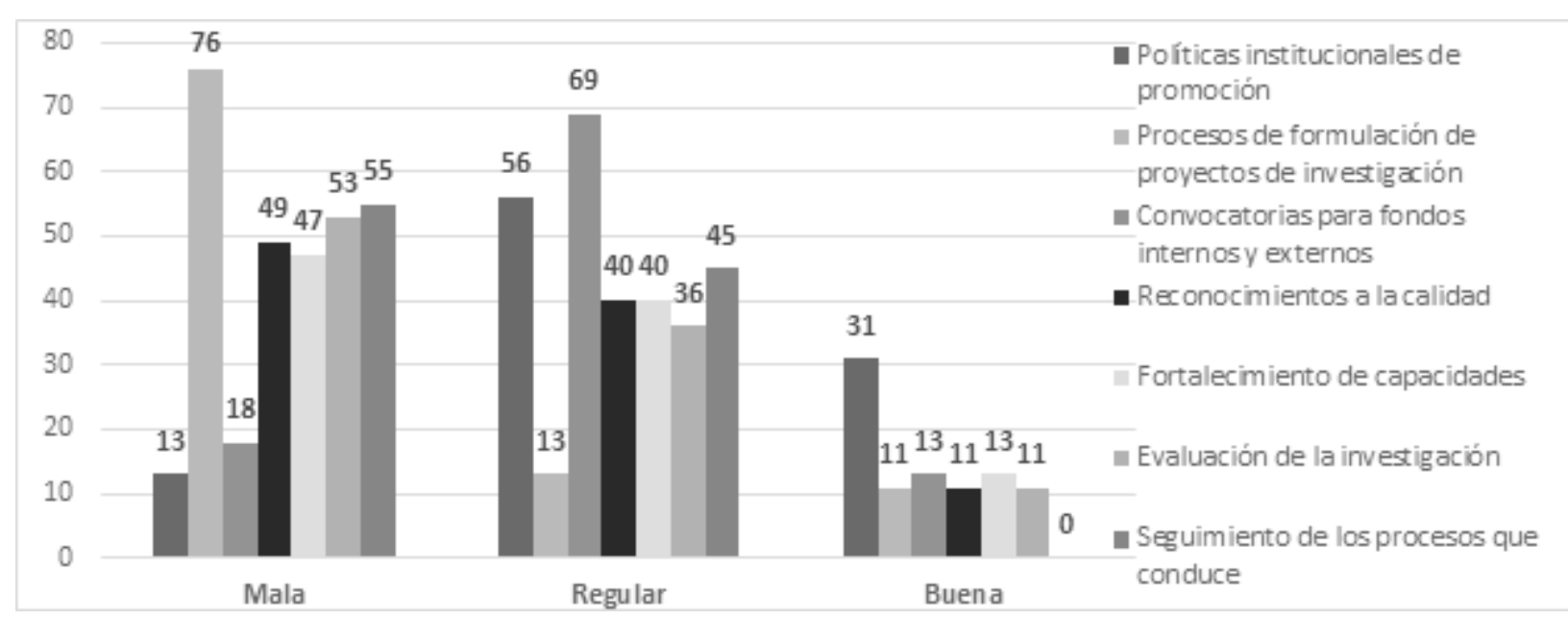

Figura 1. Opinión de los docentes sobre la forma de Gestión de la Investigación a nivel de dimensiones.

Se aprecia que, en general, existe mayor percepción de una mala gestión en 5 de las dimensiones. Dos dimensiones tienen una gestión regular y solo para una de las 7 dimensiones identificadas, los encuestados opinan que se gestiona adecuadamente. Estos resultados indican una situación preocupante en cuanto a gestión de la investigación, pues solo un $13 \%$ consideran que es "Buena", un $43 \%$ Regular y un $44 \%$ Mala. Esto se debe inter- pretar de manera cuidadosa, porque en términos generales significa que no se realiza una adecuada gestión de la investigación.

\subsection{Nivel de producción científica}

En cuanto a la gestión de la producción científica, los docentes tienen una opinión muy desfavorable, ver figura 2. Esto debido a que en la dimensión 
de accesibilidad a los resultados, el 15\% opinan que sí se gestiona, mientras que para las demás dimensiones los valores son menores al 12\%. Agregando los resultados, la situación es nuevamente preocupante, pues solo un $9 \%$ de los encuestados consideran que Sí se gestiona la Producción Científica, mientras que el $91 \%$ que No.

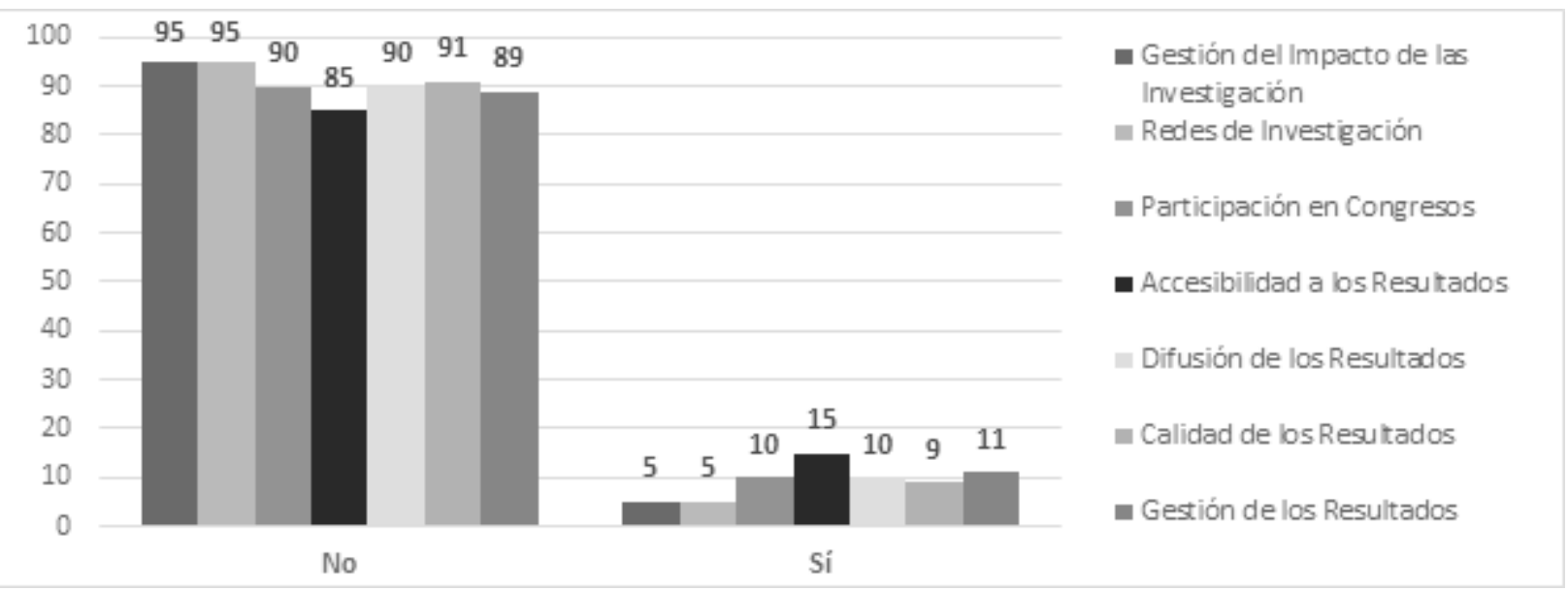

Figura 2. Opinión de los docentes sobre la gestión de la Producción Científica a nivel de dimensiones.

Lo anterior se debe interpretar de manera muy cuidadosa, porque en términos generales significa que no se realiza una adecuada gestión de la producción, que eventualmente tiene como consecuencia un deficiente nivel de producción científica, como se aprecia en la tabla 1.

Tabla 1. Producción científica de los docentes UNSM-T.

\begin{tabular}{ll}
\hline Base de datos & Valor \\
\hline Scopus & 25 \\
Scielo & 5 \\
Medline & 1 \\
Taylor And Francis & 2 \\
Willey & 1 \\
WoS & 1 \\
\hline
\end{tabular}

Los datos de la tabla 1 permiten afirmar que, a pesar de que la universidad cuenta con casi 40 años de creación, el nivel de producción científica de la misma es deficiente. La universidad no publica en mérito a su tamaño y antigüedad, por lo que se requiere de un profundo cambio estructural. Es por ello que, como parte de los resultados del estudio, en mérito de las teorías de Suárez y Díaz (2014), Acosta et al. (2017), Monteza-Arbulú (2014), y Chá-
vez-Rodríguez (2016), a continuación se propone un nuevo Modelo de Gestión de la Investigación.

\subsection{Modelo de Gestión de la Investigación}

La Universidad Nacional de San Martín - Tarapoto, UNSM-T, tiene la enorme responsabilidad de formar profesionales altamente capacitados y competentes, que al desempeñarse como tales, 
generen un cambio para bien sobre la realidad problemática identificada. Esta tarea está íntimamente ligada al desarrollo de la actividad de investigación, desarrollo e innovación, que para el caso de los estudiantes se trabaja desde el ingreso a la universidad a través de la investigación formativa (Villalba-Cuéllar \& González-Serrano, 2017). Esta formación culmina con la realización de una investigación para la obtención del título profesional, como en la propuesta de Mayta-Tristán (2016), que si bien está orientada a la carrera de medicina humana, es una opción a ser evaluada para su aplicación en otros programas de la universidad.

En cuanto a los docentes, los resultados de la investigación que desarrollan a través de los proyectos que ejecutan y asesoran, deberían culminar en artículos publicados en revistas científicas indexadas de alto impacto. Estos artículos fácilmente se pueden acceder a través de los perfiles académicos (Tena et al., 2017), especialmente el proporcionado por Google Scholar (Torres, Ruiz, \& Delgado,
2010), lo cual incrementaría la visibilidad de la producción científica de la UNSM-T.

Lo anterior coincide con Suárez y Díaz (2014), ya que entender y explicar la dinámica de la investigación universitaria y su gestión, requiere posicionarse en las prácticas que se desarrollan al interior de los recintos, trascendiendo la visión normativa contenida en los documentos formales y llenando de vida el proceso de gestión, a través de los significados que los propios actores otorgan a estos procesos.

En la figura 3 se plantea un Modelo de Gestión de la Investigación, que aplicando una visión estratégica, se espera traduzca los objetivos de la misma, en: acciones estratégicas, actividades e indicadores de producto. Estos elementos permitirán, entre otras cosas: delegar responsabilidades, designar recursos y establecer plazos para la consecución de las metas establecidas por el modelo. En los siguientes apartados se presentan las directrices del modelo.

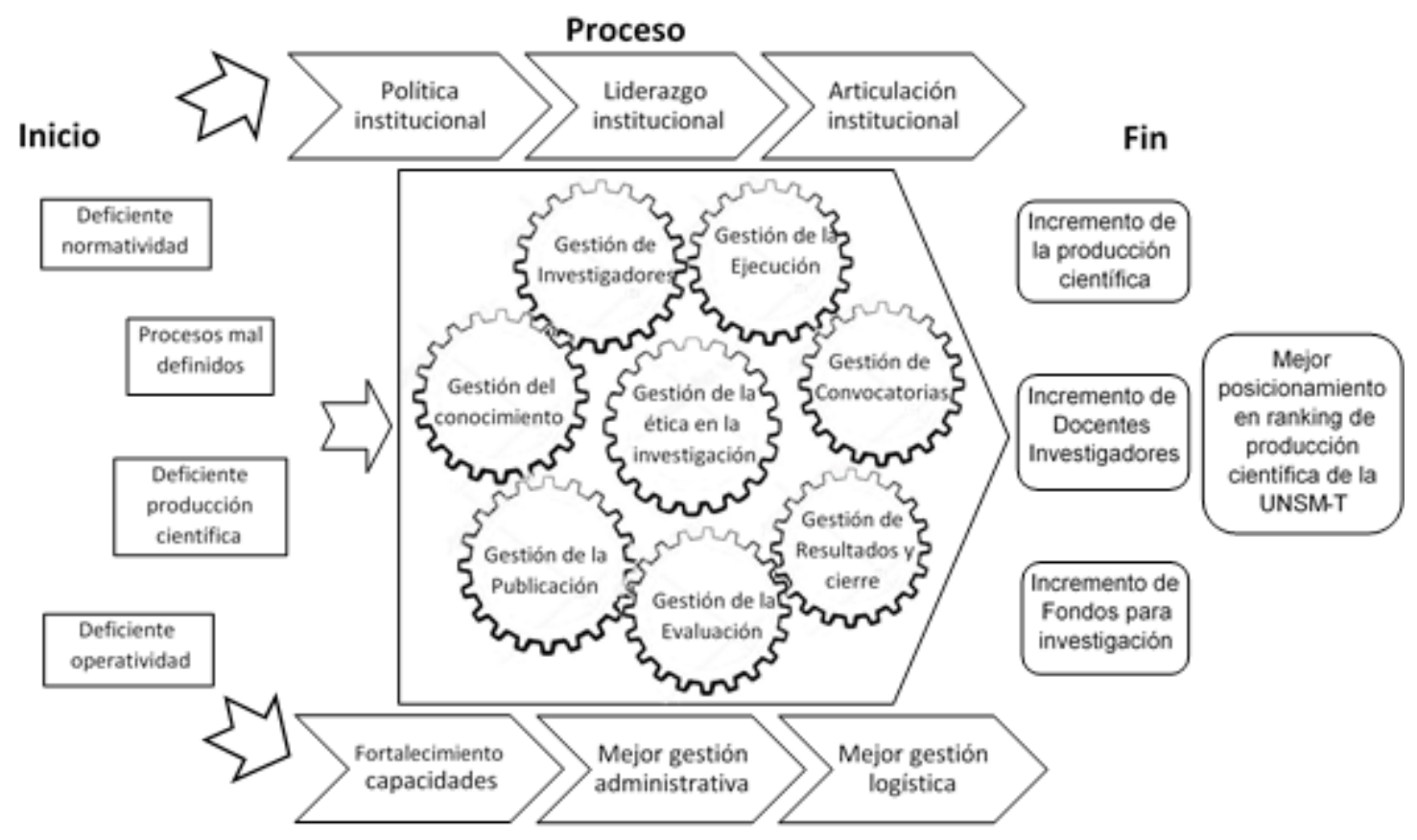

Figura 3. Modelo de Gestión de la Investigación propuesto. 


\section{Institucionalidad}

a. Políticas institucionales. Las políticas estratégicas que se establezcan, deben estar orientadas y vinculadas al eje estratégico de investigación; ya que a partir de ellas se derivan los objetivos y actividades necesarias, para asegurar la obtención de los productos que busca el modelo.

b. Liderazgo institucional. El modelo requiere además que los encargados de la dirección de las áreas estratégicas, tácticas y operativas, tengan las condiciones necesarias para asegurarse de influir positivamente en las personas, para el logro de los objetivos institucionales.

c. Articulación institucional. El modelo requiere de sobremanera, que todas las estrategias, políticas y niveles organizativos, se articulen para el logro de los objetivos y productos que busca el modelo.

\section{Gestión de la investigación}

a. Gestión de la ética en la investigación. Se debe garantizar un mayor conocimiento e interés en la implementación y el desarrollo de la ética de la investigación y la integridad científica, ya que estos son los fundamentos de la correcta ejecución de los proyectos de investigación, a fin de evitar el fraude, la fabricación de datos y el plagio. Por ello, el modelo considera a la ética el eje principal sobre el cual giran los demás engranajes propuestos.

b. Gestión de investigadores. Es necesaria una estrategia horizontal que asegure el registro, control y monitoreo de las actividades de los docentes que realicen: investigación a través de proyectos internos y externos, financiados o no financiados; asesorías de tesis de pregrado, maestría y doctorado; redacción y publicación de libros; y cualquier otra forma de generación de conocimiento. Esto permitirá evaluar qué actividades y/o recursos se les debe proporcionar, a fin de lograr la creación de los productos mínimos, como: artículos científicos, derechos de au- tor y/o cualquier otra forma de producción intelectual. Los productos permitirán posicionar adecuadamente a los docentes para lograr su categorización a nivel de universidad y a posteriori para su calificación como investigadores del Synacyt, estableciendo para ello las metas y plazos a contemplar para este trabajo.

c. Gestión de convocatorias. La búsqueda, identificación, socialización y participación en las convocatorias, se plantea como estrategia para incrementar los fondos disponibles para realizar investigación. Esto, ya que la institución no cuenta con canon y financia las investigaciones a partir de fondos de recursos ordinarios, que deberían dirigirse a financiar otras actividades académicas y administrativas de la universidad.

d. Gestión de la ejecución. Este componente permitirá que los proyectos que se estén ejecutando, presenten sus entregables en los plazos establecidos. Además, se debe garantizar, en coordinación con la parte administrativa de la universidad, la oportuna ejecución de los recursos necesarios, tanto en equipamiento, bienes y servicios, según se vayan solicitando y antes de su requerimiento en los trabajos de campo.

e. Gestión de la evaluación. La evaluación de los resultados de las investigaciones financiadas, por años, se ha basado en revisiones internas, realizadas por un equipo de profesionales muchas veces no pares académicos del tema abordado, lo cual no garantiza objetividad. El modelo propone que la evaluación sea realizada en un proceso de revisión por pares externos doble ciego, o en su defecto que se presenten productos tales como artículos científicos. En un principio, se aceptará que los artículos solamente hayan sido sometidos a las revistas. Sin embargo, para poder postularse a nuevas convocatorias, se exigirá que los artículos hayan sido aprobados para su publicación.

f. Gestión de resultados y cierre. Debe asegurarse que los productos de las investigacio- 
nes sean los esperados, debiendo entregarse en los plazos establecidos.

g. Gestión de la publicación. Se necesita de un fuerte componente de gestión de la publicación que fortalezca competencias en los docentes que realizan investigación, a través de: la ejecución de proyectos, asesoría de tesis, o el dictado de los cursos de investigación en la universidad.

h. Gestión del conocimiento. Es el componente que permitirá asegurar un proceso de retroalimentación seguro, para garantizar la transferencia del conocimiento y sobre todo permitir ampliar la experiencia institucional en la publicación de artículos científicos.

i. Gestión administrativa. Basado en Restrepo y Zabala (2016), es necesario identificar indicadores para medir su performance en las actividades relacionadas con la investigación, desarrollo e innovación. Estos permitirán la toma de decisiones orientadas al logro de los objetivos, que aumenten las ventajas competitivas, en un escenario donde lograr un empoderamiento a través del conocimiento y la experiencia, se convertirán en los activos más valiosos de la entidad.

El modelo anterior surge de las debilidades de la gestión de la investigación, detectadas en la fase diagnóstica de este trabajo. Esas debilidades impiden el correcto desarrollo de los proyectos de investigación, dificultando a su vez la generación de los productos de nuevo conocimiento. En este sentido, existe un gran trabajo por hacer, donde se articulen los engranajes institucionales relacionados con la investigación. Estos deben trabajar armoniosamente y ser dirigidos desde una perspectiva gerencial, orientada a la búsqueda de resultados, que justifiquen en principio la ejecución del gasto actual y, a mediano plazo, el incremento del presupuesto asignado a la investigación.

\section{Conclusiones}

Los resultados del estudio permiten afirmar que la percepción de la forma como se realiza la gestión de la investigación, en la Universidad Nacional de San Martín - Tarapoto, es deficiente. Esto se puede atribuir al modelo de gestión utilizado, que se caracteriza por: la deficiente incorporación de las tecnologías de información y comunicación, la poca atención al fortalecimiento de competencias en gestión de la publicación de los docentes y, en general, al poco apoyo a los aspectos logísticos y administrativos, que garanticen la eficiente ejecución de los proyectos de investigación.

Así mismo, la percepción es que no se realiza gestión de la producción científica, ya que la universidad no tiene una estrategia bibliométrica para garantizar que los resultados de las investigaciones sean publicados en artículos científicos de revistas indexadas. Operativamente hablando, por ejemplo: no se trabaja adecuadamente la gestión de la identidad digital del investigador, tampoco se fomenta el uso de referenciadores bibliográficos, y mucho menos se trabaja una estrategia de tecnología de información para el uso de los sistemas de revistas abiertas.

Esta situación ha repercutido gravemente en la visibilidad y factor de impacto de los resultados de la producción científica de los docentes, ya que a pesar de que la universidad cuenta con casi 40 años de creación, su nivel de producción científica es muy bajo, lo que le impide posicionarse adecuadamente en los rankings de producción científica.

En vista de lo anterior, se propuso un nuevo modelo de gestión de la investigación, que en mérito de la validación del juicio de expertos, permitirá una mejora o incremento de la producción científica. Para ello, el modelo se basa en tres pilares fundamentales, que son: i) institucionalidad, que busca garantizar que a nivel estratégico, táctico y ope- 
rativo se adopte el modelo para mejorar la publicación de la producción científica; ii) gestión de la investigación propiamente dicha, basada en 8 estrategias que buscan fortalecer las competencias para la publicación de artículos científicos, tomando como base la ética; y iii) gestión administrativa, que busca asegurar las condiciones logísticas y operativas para garantizar adecuados resultados de la investigación.

\section{Referencias}

Acosta, L. A., Becerra, F. A., \& Jaramillo, D. (2017). Sistema de Información Estratégica para la Gestión Universitaria en la Universidad de Otavalo, Ecuador. Formación Universitaria, 10 (2), 103-112. doi: https://doi.org/10.4067/S0718-50062017000200011

Aguillo, I. (2018). Ranking Web of Universities: More than 28000 institutions ranked. Recuperado de: http://www.webometrics.info/en/Methodology

Aguillo, I. F., Ortega, J. L., \& Fernández, M. (2008). Webometric ranking of world universities: Introduction, methodology, and future developments. Higher Education in Europe, 33 (2-3), 233-244. doi: https://doi.org/10.1080/03797720802254031

Arias-Gallegos, W. L. (2013). Crisis de la universidad en el Perú: Un problema de su naturaleza e identidad. Educación, 19, 23-39. Recuperado de: http:// revistas.unife.edu.pe/index.php/educacion/article/view/1017

British Council. (2016). La reforma del sistema universitario peruano: Internacionalización, avance, retos y oportunidades. Recuperado de: https://www. britishcouncil.pe/sites/default/files/la_reforma_ del_sistema_universitario_peruano_-_internacionalizacion_avance_retos_y_oportunidades.pdf

Cáceres-Castellanos, G. (2014). La importancia de publicar los resultados de Investigación. Revista Facultad de Ingeniería, 23 (37), 7-8. Recuperado de: http://www.scielo.org.co/scielo.php?script=sci_arttext\&pid=S0121-11292014000200001

Chávez-Rodríguez, C. (2016). Modelo de Promoción de la Investigación del Vicerrectorado de Investigación PUCP. III Congreso Nacional de Gestión de la Investigación. Lima, Perú: PUCP. Recuperado de: http://cdn02.pucp.education/investigacion/2016/11/10173224/Ponencia-magistral-2-Mg--Carlos-Chavez.pdf

Cuencia, R. (2014). Aula Magna 2014: "La Universidad del Siglo XXI" (Tercer Panel). Lima: PUCP. Recuperado de: http://vicerrectorado.pucp.edu.pe/ academico/noticias/aula-magna-2014-la-universidad-del-siglo-xxi-tercer-panel/

Forero-Jiménez, M. Y. (2019). Modelo de responsabilidad social universitaria: una propuesta para las instituciones colombianas. Revista de Investigación, Desarrollo e Innovación, 9 (2), 249-260. doi: 10.19053/20278306.v9.n2.2019.9160

Gestion. (2015). Sunedu: Solo cinco universidades han cumplido con elegir a sus nuevas autoridades. Recuperado de: https://gestion.pe/economia/ sunedu-cinco-universidades-han-cumplido-elegir-nuevas-autoridades-84545

Jiménez-Tello, M. del P. (2007). Auditoría Universitaria y Calidad. Universidad Autónoma de Barcelona. Recuperado de: https://www.tdx.cat/bitstream/ handle/10803/5233/pjt1de1.pdf

Lavalle, C., \& de Nicolas, V. L. (2017). Peru and its new challenge in higher education: Towards a research university. PLOS ONE, 12 (8), 1-12. doi: https://doi.org/10.1371/journal.pone.0182631

Ley 30220. (2014). Ley Universitaria. Lima. Recuperado de: https://www.sunedu.gob.pe/wp-content/uploads/2017/04/Ley-universitaria-30220. pdf 
Martínez-de Ita, M. E., Piñero, F. J., \& Figueroa-Delgado, S. A. (2013). El papel de la universidad en el desarrollo. Puebla, México: Benemérita Universidad Autónoma de Puebla. Recuperado de: http://biblioteca.clacso.edu.ar/clacso/se/20140211121020/ universidad.pdf

Mayta-Tristán, P. (2016). Tesis en formato de artículo científico: oportunidad para incrementar la producción científica universitaria. Acta Médica Peruana, 33 (2), 95-98. Recuperado de: $\quad$ http://www.scielo.org.pe/scielo.php?pi$\mathrm{d}=$ S1728-59172016000200001\&script=sci_arttext\&tlng=pt

Monteza-Arbulú, C. (2014). Modelo de gestión de la investigación y nivel de desarrollo de la investigación universitaria. UCV-Hacer. Revista de Investigación y Cultura, 3 (2). Recuperado de: http://www. redalyc.org/pdf/5217/521751976008.pdf

Ortiz-Torres, B. (2018). Impacto social de la Investigación. IV Congreso Nacional de Gestión de la Investigación, 25 a 27 de Septiembre de 2017. Lima, Perú: Pontificia Universidad Católica del Perú. Recuperado de: http://investigacion.pucp.edu.pe/

Restrepo, G., \& Zabala, D. (2016). Indicadores de gestión para proyectos de investigación y extensión en instituciones de Educación Superior. Revista Ciencias Estratégicas, 24 (36), 451-461. doi: https://doi.org/10.18566/rces.v24n36.a13

SINEACE. (2013). Educación Superior en el Perú: Retos para el Aseguramiento de la Calidad Estudio del SINEACE, encargado a los especialistas. (Cesar La Serna Venegas, Ed.) (Primera). Lima, Perú: SINEACE. Recuperado de https://www.sineace.gob.pe/ wp-content/uploads/2013/08/Retos-para-el-aseguramiento-de-la-calidad.pdf

Suárez, W., \& Díaz, J. (2014). Gestión de la investigación en la universidad pública venezolana. Telos. Revista de Estudios Interdisciplinarios En Ciencias Sociales, 16 (1), 135-156. Recuperado de: https:// biblat.unam.mx/es/revista/telos-revista-de-estudios-interdisciplinarios-en-ciencias-sociales/articulo/gestion-de-la-investigacion-en-la-universidad-publica-venezolana

SUNEDU. (2018). Informe Bienal sobre la Realidad Universitaria. Lima, Perú: Impresión Arte Perú S.A.C. Recuperado de: https://www.sunedu.gob. pe/informe-bienal-sobre-realidad-universitaria/

Tena, M., García, A., Holgado, A., Merlo, J., \& Peñalvo, F. (2017). Diseño de un plan de visibilidad científica e identidad digital para los investigadores de la Universidad de Guadalajara (México). Ibersid, 11 (1), 83-92. Recuperado de: https://gredos.usal.es/ jspui/bitstream/10366/133378/1/4337-6437-1-PB. pdf

Times Higher Education. (2019). World University Rankings 2019. Recuperado de: https://www. timeshighereducation.com/world-university-rankings/2019/world-ranking\#!/page/0/length/25/ locations/PE/sort_by/rank/sort_order/asc/cols/ stats

Torres, D., Ruiz, R., \& Delgado, E. (2010). Google Scholar: ¿una herramienta para la evaluación de la ciencia? Anuario ThinkEPI, 4 (0), 254-257. Recuperado de: https://recyt.fecyt.es/index.php/ ThinkEPI/article/view/31269/16618

Universidad Politécnica de Cataluña, \& Centro Interuniversitario de Desarrollo. (2007). Acreditación y dirección estratégica para la calidad en las universidades. Santiago de Chile: Universidad Politécnica de Cataluña. Recuperado de: http://www.upch. edu.pe/rector/dugec/images/files/biblioteca/36. PDF

Valles-Coral, M. A., \& Rengifo-Arias, M. A. (2018). Estrategia de TI para el control de indicadores de gestión en la investigación en la UNSM-T, 2017. Tarapoto: Universidad Nacional de San Martín - Tarapoto. Recuperado de: http://repositorio.unsm. edu.pe/bitstream/handle/11458/2913/INVESTI- 
GACION-MiguelAngelVallesCoral \%26 Miguel Angel Rengifo Arias.pdf?sequence=1\&isAllowed=y

Vargas, J. Y. (2019). En investigación aún hay mucho por proponer. Horizonte de La Ciencia, 9 (16). Recuperado de: http://revistas.uncp.edu.pe/index. php/horizontedelaciencia/article/view/480/371
Villalba-Cuéllar, J. C., \& González-Serrano, A. (2017). La importancia de los semilleros de investigación. Prolegómenos, 20 (39), 9-10. Recuperado de: http://www.scielo.org.co/scielo.php?script=sci_arttext\&pid=S0121-182X2017000100001 